\title{
The Influence of Rhizosphere Fungal Diversity and Complex Community Structure to Wheat Root Rot Disease
}

\author{
Xuejiang Zhang ${ }^{1}$, Heyun Wang ${ }^{2}$, Gavin $\mathrm{Ash}^{3}$, Dazhao $\mathrm{Yu}^{1}$, Hua Wang ${ }^{1,{ }^{*}}$
}

The full names, email addresses and institutional addresses for all authors

${ }^{1}$ Xuejiang Zhang

zhangxuejiang1976@126.com

Institute of Plant Protection and Soil \& Fertilizer, Hubei Academy of Agricultural

Sciences/Key Laboratory of Integrated Pest Management on Crops in Central China, Ministry of

Agriculture. P. R. China/Hubei Key Laboratory of Crop Disease, Insect Pests and Weeds Control, Wuhan 430064, P. R. China.

${ }^{2}$ Heyun Wang

heyunwang2006@163.com

HuBei University of Technology, Wuhan 430068, P. R. China.

${ }^{3}$ Gavin Ash

Gavin.Ash@usq.edu.au

The Centre for Crop Health, University of Southern Queensland, Toowoomba QLD 4350, Australia.

${ }^{1}$ Dazhao Yu

"Corresponding author: wanghua4@,163.com

${ }^{2}$ Heyun Wang contributed equally to this work.

${ }^{1}$ Institute of Plant Protection and Soil \& Fertilizer, Hubei Academy of Agricultural Sciences, Nanhu Road 6, Wuhan 430064, China.

Full list of author information is available at the end of the article. 
dazhaoyu1956@sina.com.cn

Institute of Plant Protection and Soil \& Fertilizer, Hubei Academy of Agricultural

Sciences/Key Laboratory of Integrated Pest Management on Crops in Central China, Ministry of

Agriculture. P. R. China/Hubei Key Laboratory of Crop Disease, Insect Pests and Weeds Control, Wuhan 430064, P. R. China.

\title{
Corresponding author:
}

${ }^{1, *}$ Hua Wang

wanghua4@163.com

Institute of Plant Protection and Soil \& Fertilizer, Hubei Academy of Agricultural

Sciences/Key Laboratory of Integrated Pest Management on Crops in Central China, Ministry of Agriculture. P. R. China/Hubei Key Laboratory of Crop Disease, Insect Pests and Weeds Control, Wuhan 430064, P. R. China.

\begin{abstract}
Background: Wheat root rot disease due to soil-borne fungal pathogens leads to tremendous yield losses worth billions of dollars worldwide every year. It is very important to study the relationship between rhizosphere fungal diversity and wheat roots to understand the occurrence and development of wheat root rot disease.
\end{abstract}

Results: A significant difference in fungal diversity was observed between the diseased and healthy groups in the heading stage, but the trend was the opposite in the filling stage. The abundance of most genera with high richness decreased significantly from the heading to the filling stage in the diseased groups; the richness of approximately one-third of all genera remained unchanged, and only a few low-richness genera, such as Fusarium and Ceratobasidium, had a very significant increase from the heading to the filling stage. In the healthy groups, the abundance of most genera increased significantly from the heading to the filling stage; the abundance of some genera did not change markedly, or the abundance of very few genera increased significantly. Physical and chemical soil indicators showed that low soil $\mathrm{pH}$ and density, increases in ammonium nitrogen, nitrate nitrogen and total nitrogen contributed to the occurrence of wheat root rot disease.

Conclusions: Our results revealed that in the early stages of disease, highly diverse rhizosphere fungi and a complex community structure can easily cause wheat root rot disease. The existence of pathogenic fungi is a necessary condition for wheat root rot disease, but the richness of pathogenic 
fungi is not necessarily important. The increases in ammonium nitrogen, nitrate nitrogen and total nitrogen contributed to the occurrence of wheat root rot disease. Low soil $\mathrm{pH}$ and soil density are beneficial to the occurrence of wheat root rot disease.

Keywords: Rhizosphere, Fungal diversity, Community structure, Wheat root rot disease

\section{Introduction}

Soil microbial diversity is important to sustainable agriculture because microbes mediate many processes that are essential to the agricultural productivity of soil (1). However, to meet the food demand of an increasing population, intensive agricultural practices and the cultivation of exhaustive crops have destroyed soil structure and ignored the biological potential of roots or rhizospheres to efficiently mobilize and acquire soil nutrients (2-8). As such, soils have very low biological activity, and plants growing in these soils are predisposed to soil-borne pathogens (9-12).

With the destruction of soil structure, the degradation of soil and the increase in soil-borne pathogens, wheat, as one of the three major staple foods in the world, grown in Asia (China), Australia, Europe, North America, and South America, is vulnerable to attack by a complex of root pathogens, which results in tremendous yield losses (13). Annual losses in wheat industries due to soil-borne fungal pathogens amount to over billions of dollars worldwide (14-16). All cultivars of wheat are attacked by several soil-borne fungal pathogens that cause root diseases (15). The primary fungal pathogens include the following: Fusarium culmorum, Fusarium pseudograminearum, Gaeumannomyces graminis var. Tritici, Bipolaris sorokiniana, and Alternaria spp. in Ascomycota; Rhizoctonia, Rhizopus oryzae, Rhizoctonia solani and Penicillium spp. in Basidiomycota; Pythium spp. in Oomycota; and Curvularia spp. in Deuteromycota $(10,17)$. Root diseases are difficult to control because these soil-borne fungi are ubiquitous, the pathogens often occur as a complex (15, 18), and they can easily survive on infected plant debris or form durable chlamydospores in the soil with or in the absence of growing hosts and can outgrow or evade plant defenses $(10,19)$. There are no resistant varieties among adapted cultivars of wheat (20-21) and no chemical controls, although certain seed treatments can provide some early benefits to seedling health (21-22).

Therefore, it is very important to study the relationship between soil microbial diversity and plants to understand the occurrence and development of crop root rot. Plants depend on the ability of roots to communicate with rhizosphere microorganisms through signaling pathways, creating a connection between plants and microorganisms (23-25). The composition of the rhizosphere microbiota can negatively or positively influence plant traits such as stress tolerance, health, 
development, and productivity (26-28). The plant, in turn, cultivates the structural and functional diversity of microbial communities in the rhizosphere by adjusting soil $\mathrm{pH}$, releasing secondary metabolites (e.g., pyrones, sesquiterpenes) into the rhizosphere $(25,29-30)$, reducing competition for beneficial microbes, and providing an energy source, mostly in the carbon-rich rhizosphere (4, 21). Soil-plant-microbial health must remain in equilibrium to maintain sustainable agricultural practices $(25,31-32)$. Under unfavorable conditions, some fungi can cause plant diseases and sometimes even total loss of crop yields (28).

In addition to agrochemicals (33), fertilization (8, 34), soil types (35-36), tillage (1) and crop rotation (9-10), which can influence rhizosphere microorganisms, the structure and function of rhizosphere microflora may also be affected by the plant physiology of different plant genotypes (36-37) and may also fluctuate among the vegetation stages of the same plant genotype $(36,38)$. Plant growth stage influences root physiology and changes the quality and quantity of root exudates; consequently, these changes select for different root-associated microorganisms at different growth stages (39-41). The purpose of this study was to study the diversity of fungi, the variation in community structure and the trends in the microbial species in the rhizosphere of healthy and diseased wheat roots at different heading and filling stages of wheat growth. Additionally, by combining these results with the physical and chemical properties of soil, the possible causes of wheat root rot disease were revealed, providing an important theoretical basis and practice for the improved control of wheat root rot disease.

\section{Materials and methods}

\subsection{Rhizosphere samples}

From 2015 to 2016, we conducted a continuous follow-up survey on land planted with wheat at Xiangyang Original Farm in Hubei Province, China, and found that the wheat root rot disease at this farm was serious (Fig 1 A). In this study, wheat root samples were collected in early April (heading stage) and early May (filling stage) of 2017 (Fig 1 B). The sampling method consisted of first investigating the occurrence of wheat root rot, selecting weak seedlings and sampling the brown parts of the root as the diseased wheat rhizosphere. Areas with diseased wheat and healthy wheat (the diameter of each area was not more than $10 \mathrm{~cm}$ ) were marked by inserting cards at fixed points at least 10 meters apart. Then, plants were sampled at 20 points, and 5 wheat plants were sampled at each point. The roots of the whole plant and the soil on the roots were collected. The majority of soil on the roots was shaken off, and samples were collected for the determination of physical and chemical soil properties. The roots of five wheat plants were placed in a sealed pocket and quickly stored in a dry-ice box for dry-ice preservation. After all the samples were collected, they were 
immediately brought to the laboratory and stored in a cryogenic refrigerator for future use. In May, the samples were collected from the areas labeled in April. The ears of diseased wheat in this area had a clear white color, and these plants had markedly brown, black or yellow roots. Five soil samples from the diseased wheat root and five soil samples from the healthy wheat root were collected in May. The sampling method was the same as that for the previous collection. According to the cards inserted in the soil in April, the 20 samples collected in April, which were identical to the samples collected in May, were removed from the cryogenic refrigerator to analyze the diversity of fungi in the wheat rhizosphere.

\subsection{Extraction and PCR amplification of total genomic DNA from rhizosphere fungi}

The soil attached to the root was brushed off, a 0.1-g soil sample was accurately weighed, and the total genomic DNA from all samples was extracted according to the instructions provided with an E.Z.N.A. ${ }^{\circledR}$ Stool DNA Kit (Omega Biotek, Norcross, GA, U.S.). Total DNA was detected by $1 \%$ agarose gel electrophoresis, and the purity and concentration of DNA were determined by spectrophotometry. All DNA samples were stored in a refrigerator at $-20^{\circ} \mathrm{C}$. Fungal diversity was determined by amplifying the ITS1 region using the CS1_ITS1F and CS2_ITS2R primer sets for fungi. The primer sequences were ITS1F 5'- CTTGGTCATTTAGAGGAAGTAA-3' and ITS2R 5'GCTGCGTTCTTCATCGATGC-3', and the amplification conditions were predenaturation at $95^{\circ} \mathrm{C}$ for $5 \mathrm{~min}, 27 \mathrm{cycles}$ of $95^{\circ} \mathrm{C}$ for $30 \mathrm{~s}, 55^{\circ} \mathrm{C}$ for $30 \mathrm{~s}$, and $72^{\circ} \mathrm{C}$ for $45 \mathrm{~s}$, and elongation at $72^{\circ} \mathrm{C}$ for 5 min. Three replicates of the PCR were performed, and a $20-\mu \mathrm{L}$ reaction system $(4 \mu \mathrm{L}$ of $5 \times$ FastPfu buffer solution, $2 \mu \mathrm{L}$ of $2.5 \mathrm{mM}$ dNTPs, $0.8 \mu \mathrm{L}$ of primer $(5 \mu \mathrm{M}), 0.4 \mu \mathrm{L}$ of FastPfu polymerase and $10 \mathrm{ng}$ of fungal total genomic DNA) was used.

\subsection{Illumina HiSeq2500 sequencing}

The PCR amplification product was recovered by $2 \%$ agarose gel electrophoresis followed by the use of an AxyPrep DNA gel Recovery Kit (Axygen Biosciences, Union City, CA, U.S.), and the quantitative analysis was carried out using a QuantiFluor ${ }^{\circledR}-\mathrm{ST}$ (Promega, U.S.). Two-terminal sequencing was carried out using a HiSeq2500 platform after equally mixing the purified products. The original read data were deposited in the NCBI Sequence Read Archive (Accession number: SUB5829339).

\subsection{Data processing}

Using Perl script, the offline data were divided into different sample data groups according to 
barcode sequences, and the barcode sequence and PCR-amplified primer sequence were truncated. Then, the divided data were spliced with FLASH to connect the reads of each sample, and the splicing sequence was the original tag data (raw tags). The raw tags obtained by splicing were strictly filtered to obtain high-quality tag data (clean tags). For tag quality control, the following operations in QIIME were used: a) tag intercept, truncation of raw tags from the first low-quality base site with consecutive low-quality bases (default mass threshold was $\leq 3$ ) to the set length (default length was 3); b) tag length filtering, tag data sets obtained by tag intercepts were further filtered to remove tags with continuous high-quality bases of less than $75 \%$ of the tag length; c) chimera filtration. After the above processing steps, the tag sequences were compared to sequences in databases (Gold database, http:/driver5.com/uchime_download.html; UCHIME algorithm, http://www.drive5.com/usearch/manual/uchime_algo.html), the chimera sequence was detected, and the chimera sequence was finally removed to obtain the final valid data (effective tags). The sequencing depth was more than 30,000 original reads per library.

\subsection{OTU and species community analysis}

UPARSE software (Uparse v7.0.1001, (http://drive5.com/uparse/)) was used to cluster the effective tag sequences of all samples with $97 \%$ and $95 \%$ consistency to form operational taxonomic units (OTUs). The representative sequence of each OTU was identified, the assin_taxonomy.py script (http://qiime.org/scripts/assign_taxonomy.html) in QIIME was used, and RDP (16S, http://rdp.come.msu.edu/), Silva (18S, http://www.arb-silva.de) and Unite (ITS) were used to compare the data with the database to obtain species annotation information. Then, the annotated chloroplast or mitochondrial genes (16S amplifiers) and the OTUs and tags that could not be annotated at the boundary level were removed (confidence threshold was above 0.8 by default). Then, the OTUs and tags annotated as chloroplasts or mitochondria (16S amplifiers) and those that could not be annotated at the kingdom level were removed. Finally, the effective tag data, low-frequency tag data, annotated tag data, chloroplast and mitochondrial data and OTU data obtained from each sample were counted by a script. Additionally, we used R software to calculate the annotation ratio of OTUs and each taxonomic level and the relative abundance of the species in each taxonomic level.

Based on the above valid OTU data, the following evolutionary analysis was carried out: a) evolutionary relationships and relative abundance information of species systems based on OTU data in samples were determined, and species annotation results for a single sample were visualized using KRONA software (http://sourceforge.net/projects/krona); b) related genera were selected, a phylogenetic tree for the OTUs of these genera was constructed (QIIME software package: make_phylogeny.py (http://qiime.org/scripts/make_phylogeny.html)), and the systematic evolutionary relationship was displayed by combining the relative abundance of OTUs and the 
reliability of annotation using a Perl script; and c) local Perl scripts were used to select the dedicated OTUs for intrasample and intersample phylogenetic analysis and to compare relative abundance.

To analyze the community structure of species, relative abundance thermograms were plotted at the OTU level and the genera level by R software. Cluster analysis and principal coordinate analysis (PCoA) were also carried out to compare samples.

\section{Results}

\subsection{Fungal diversity in the rhizosphere}

According to the diversity index, the community richness and diversity of the diseased groups were higher than those of the healthy groups in the heading stage, and there was a significant difference in community diversity between the diseased group and the healthy group (Shannon index: $P<0.01$ ) (Supplemental table 1). The high community richness and diversity created conditions for subsequent diseases. However, the community richness and diversity of the disease group and the healthy group in the filling stage were higher than those of the disease group and the healthy group in the heading stage, and there was a significant difference between the disease group and the healthy group in the heading stage (Sobs index: $P<0.05$; Shannon index: $P<0.05$ ). The community richness and diversity of the healthy group in the filling stage were higher than those of the diseased group in the filling stage.

\subsection{Community structure of fungi in the rhizosphere}

There were 1393 OTUs distributed among 7 phyla, 346 genera and 549 species. The seven phyla were Ascomycota, Basidiomycota, Zygomycota, Chytridiomycota, Glomeromycota, Blastocladiomycota and unclassified fungi (Fig 2 A). Among these phyla, Ascomycota was the most abundant. The richness values were $65.48 \%$ and $67.61 \%$ and were $72.57 \%$ and $57.35 \%$ in healthy groups 4 and 5 (H4 and H5, respectively) and diseased groups 4 and 5 (D4 and D5, respectively), respectively, but there was no significant difference among the populations. The other three phyla showed very interesting results. Basidiomycota had the highest abundance in population $\mathrm{H} 4$ in the heading stage $(25.3 \%)$. However, in the D4, D5 and H5 populations, the abundance was significantly decreased to $12.36 \%, 11.16 \%$ and $14.7 \%$, respectively. Significant differences were found between populations $\mathrm{H} 4$ and D4 and populations D5 and H5 $(\mathrm{P}<0.01)$. The abundance of Zygomycota in populations $\mathrm{H} 4$ and D4 was low in the heading stage $(3.16 \%$ and $2.44 \%$, respectively), but suddenly increased to $9.87 \%$ (H5) and $7.42 \%$ (D5) in the filling stage. However, the trends in the abundance of Chytridiomycota and Zygomycota were almost the opposite. The abundance of Chytridiomycota in the heading stage groups was $4.52 \%(\mathrm{H} 4)$ and $7.18 \%$ (D4), and 
there was a significant difference between these two groups. The abundance of Chytridiomycota in population D4, a diseased group, was much higher than that in the healthy groups. By May, the abundance of Chytridiomycota in population H5 decreased sharply to $1.37 \%$, while that of population D5 decreased to only $4.12 \%$.

Excluding unclassified fungi, all fungi with richness greater than $1 \%$ in a single group were counted (Table 2). There are 30 genera in the four groups belonging to Ascomycota, Basidiomycota, Chytridiomycota and Zygomycota. Among these genera, Chytridiomycota and Zygomycota had only one genus, Olpidiaster and Portierella, respectively. Basidiomycota had seven genera, and Ascomycota had the largest distribution, with 21 genera (Fig 2 B and C, Supplemental table 2). In the heading stage, the abundance of the most highly abundant genera in population D4 was significantly decreased in population D5 at the filling stage; however, the abundance of Mortierella significantly increased, the abundance of one-third of the genera remained almost unchanged, and the abundance of a few low abundance genera, such as Fusarium and Ceratobasidium, significantly increased in the diseased population over time. Seven genera with high richness were found in the $\mathrm{H} 4$ population at the heading stage, and the richness of these genera was significantly increased in the $\mathrm{H} 5$ population at the grain filling stage; among these genera, the variation in the richness of Microdochium, Mycosphaerella, Scytalidium, Acremonium and Olpidiaster from the H4 population to the $\mathrm{H} 5$ population was the opposite of the change in abundance from the $\mathrm{D} 4$ population to the D5 population. The richness of 13 genera did not markedly change, and the richness of 4 low-richness genera was significantly increased at the grain filling stage compared with the heading stage. In general, the following six genera showed no change in richness from the heading stage to the grain filling stage, between the H5 and D5 populations and the H4 and D4 populations, respectively, Alternaria, Sarocladium, Gibberella, Cladosporium, Neosetophoma and Cystofilobasidium. The genera with significant or extremely significant differences in richness among the groups were Alternaria, Mortierella, Cryptococcus, Apodus, Epicoccum, Scytalidium and Chaetomium.

A Venn diagram of genera with richness greater than $1 \%$ was constructed (Fig 2 D). There were 153 shared genera, 35 endemic genera that appeared most frequently in the healthy group in the filling stage and 7 and 10 genera in the heading stage and filling stage, respectively. The results showed that the root exudates of diseased plants specifically promoted the growth of microbial fungi, while the rhizosphere of healthy plants was more suitable for the growth of various fungi. An increased diversity of soil microorganisms promoted healthy plant growth.

\subsection{PCoA of populations}

The PCoA of fungal community structure in different samples based on OTU revealed that the 
populations could be divided into two groups: the healthy group and the diseased group (Figure $3 \mathrm{~A}$ and B). There was no overlap between the two groups, which indicated that the samples were consistent with the expectation and that the microbial community structures of the diseased group and the healthy group were quite different. The diseased group at the heading stage was significantly separated from the diseased group at the filling stage, and the microbial community structure was quite different. The healthy group at the heading stage and the healthy group at the filling stage were also clearly separated, and the microbial community structure was quite different. In addition, according to the flora classification, all rhizosphere fungi were divided into two types (Fig 3 C): type one included the diseased group, and type two included the healthy group. Only sample H5-5 was assigned to type one.

\subsection{Effects of physical and chemical soil properties on rhizosphere fungi}

The main physical and chemical properties of soil measured were total phosphorus (TP), ammonium nitrogen $\left(\mathrm{NH}_{4}\right)$, nitrate nitrogen $\left(\mathrm{NO}_{3}\right)$, total nitrogen $(\mathrm{TN})$, $\mathrm{pH}$, soil density $(\mathrm{SD})$, total carbon (TC) and the soil dry-humidity ratio (DHR) (Table 3). TP and TC decreased and $\mathrm{NH}_{4}$ increased from the heading stage to the filling stage. Moreover, the $\mathrm{NO}_{3}$ and $\mathrm{TN}$ of the diseased group were higher than those of the healthy group, and the $\mathrm{pH}$ value and $\mathrm{SD}$ of the diseased group were lower than those of the healthy group. Heatmap cluster analysis based on Spearman correlation coefficients for the 30 genera with the most richness and these physical and chemical indicators was performed (Fig 4). The graph shows that $\mathrm{pH}$ and SD have the greatest influence on microbial abundance, and the influence of these factors is in the same direction. One-third of the genera were positively or significantly positively correlated with both $\mathrm{pH}$ and SD, and one-third were negatively or significantly negatively correlated with both $\mathrm{pH}$ and $\mathrm{SD}$. The effects of $\mathrm{NO}_{3}$ and $\mathrm{TN}$ on microbial richness were similar, but the impact of these factors on microbial species richness was almost the opposite of that of $\mathrm{pH}$ and SD. Soil DHR and TP had moderate effects on microbial richness. The physical and chemical properties of soil with the least influence on microbial abundance were $\mathrm{NH}_{4}$ and TC.

\section{Discussion}

Microbial diversity in soil is an important factor that determines soil health and is considered one of the main contributors to soil suppressiveness $(28,42)$. The rhizosphere is one of the most complex environments; rhizospheres are influenced by plant roots and are an active microhabitat where plant roots and microbes interact $(28,43-44)$. Throughout the heading and filling stages of wheat, the fungi in the rhizosphere showed high diversity. The diversity of soil fungi at the grain filling stage was significantly higher than that at the heading stage. It is already well known that most crops can 
significantly benefit from establishing associations with diverse soil microbes (25). Plants stimulate or inhibit the growth of specific rhizosphere microorganisms by releasing secondary metabolites into the rhizosphere $(25,29-30)$. In the rhizosphere of wheat with root rot disease, the interaction between wheat and microorganisms was intense, and the diversity and richness of the fungal community in the rhizosphere of the diseased group were significantly higher than that of the healthy group, creating conditions for the occurrence of wheat root rot. However, at the filling stage, wheat roots were clearly diseased due to infection by pathogenic fungi. During this process, the metabolites secreted by wheat roots tend to be monotonous. As a result, the fungi in the rhizosphere of diseased plants showed lower community diversity and richness than those in the rhizosphere of healthy plants. The interaction between rhizosphere fungi and wheat roots in the healthy group reached an optimal balance during the filling stage and then became mutualistic, which was favorable for the healthy growth of the fungi and wheat. Therefore, in the filling stage, the diversity and richness of fungi in the rhizosphere were higher in the healthy group than in the diseased group.

The richness change analysis at the phylum level revealed that the phylum with the highest richness was Ascomycota, which consisted of more than $60 \%$ of all healthy and diseased groups, and there was no significant difference among groups. However, the main pathogenic fungi that cause wheat root rot disease, such as F. culmorum, F. pseudograminearum, G. graminis var. Tritici, B. sorokiniana and Alternaria spp., are in this phylum. There are also some fungi that cause wheat root rot in the phylum Basidiomycota, such as Rhizoctonia, R. oryzae, R. solani and Penicillium spp. This phylum showed high richness in only the healthy group at the heading stage (25.3\%), but the richness of this phylum was significantly decreased in the other groups $(<14 \%)$. Analysis of the abundance of these two phyla shows that the impact of the fungi that cause wheat root rot disease is not due to phylum-level abundance. The richness of two other phyla, Zygomycota and Chytridiomycota, which contain almost no root rot fungi, showed notable differences between the heading and filling stages in the healthy group but showed no significant differences between these stages in the diseased groups. This change in abundance may contribute to the healthy growth of wheat roots or may be a cofactor in the occurrence of wheat root rot disease.

Relative to the changes at the phylum level, levels of richness at the genera level varied greatly. The abundance of most genera with high richness decreased significantly from the heading stage to the filling stage in the diseased groups; the richness of approximately one-third of all genera remained unchanged, and only very few low-richness genera, such as Fusarium and Ceratobasidium, had a very significant increase in richness over time. In the healthy group, the abundance of most genera increased significantly from the heading stage to the filling stage, except for some genera whose abundance did not markedly change or very few genera whose abundance increased significantly. This result also shows that the interactions between wheat roots and rhizosphere fungi must achieve a balance. If this balance is lost, the wheat roots will become diseased. From the analysis of the endemic genera in each group, we also found that there were 35 endemic genera in the healthy group, 
which was many more than the approximately 10 endemic genera in the diseased group, indicating that the healthy growth of wheat roots can promote plant growth and suppress disease through various activities that prevent infection by pathogens. Therefore, the rhizosphere microorganisms show improved growth, and the microbial diversity and community richness are also significantly increased in the healthy plants compared with the diseased plants.

Fungi and fungus-like organisms form one of the most diverse groups of eukaryotes and represent an essential functional component of soil microbial communities $(28,45)$. Under unfavorable conditions, some fungi can cause plant diseases and sometimes even the total loss of crop yields. In many instances, these diseases are caused by a complex of fungal species (28). Only Alternaria and Fusarium, genera with richness values greater than one percent, have been documented to cause wheat root rot, but the abundance of these two genera is not high. Although the abundance of Alternaria in the diseased groups was significantly higher than that in the healthy groups, there was no significant change between the heading stage and filling stage. The abundance of Fusarium in the filling stage was significantly higher than that in the heading stage. Considering the abundance of these two pathogens, the richness level of a fungus does not indicate whether it can lead to wheat root rot. In addition, other pathogenic fungal genera mentioned in the literature were determined to have less than one percent abundance. Therefore, we can speculate that as long as these fungal genera exist in the rhizosphere, they can lead to wheat root rot disease, regardless of the abundance. However, whether a fungus can induce root rot disease depends on the result of the interaction between wheat root and rhizosphere microorganisms, which is also closely related to seasonal climatic conditions.

Differences in the fungal community structure among groups can clearly demonstrate the heterogeneity of each group, thus showing why there are so many differences in the rhizosphere fungi of the diseased groups and the healthy groups. PCoA clustering analysis clearly clustered the four groups into two groups: the healthy group and the diseased group. However, the healthy group and the diseased group were each clearly separated into the heading stage and grouting stage. In other words, the healthy group and the diseased group had significant differences in fungal community structure, and the difference in the fungal community structure of the heading stage and the grouting stage was significant. In addition, all rhizosphere fungi were divided into two types according to flora classification: one type included only the healthy group, and the other type included only the diseased group. When wheat roots were attacked by microorganisms and developed root disease, the structure of the rhizosphere fungal community was markedly changed. To differentiate between the fungi located very close to the epidermis in the root zone and to protect against the invasion of heterogeneous microbes, plants continuously secrete signaling molecules, which allows for the development of pathogenic, associative, symbiotic, or naturalistic relationships between microbes and the plant $(25,46)$. 
At the heading stage, the root is slightly diseased, and the interaction between wheat roots and rhizosphere fungi is the most intense, resulting in marked heterogeneity of the soil environment and inducing a high level of rhizosphere fungal diversity and a complex community structure. However, at the filling stage, the wheat roots are completely diseased. At this time, the soil environment is stable, and the material secreted by wheat roots is relatively limited, which leads to a decrease in the microbial diversity of the rhizosphere and a relatively simple community structure. Soil-plant-microbial health must be maintained at an equilibrium to maintain sustainable agricultural practices $(25,31-32)$. At the heading stage, the antagonistic interaction between healthy wheat roots and rhizosphere fungi reaches a balance and promotes the healthy growth of wheat and fungi. By the filling stage, the ecological environment, such as temperature, humidity and other factors, is improved, the rhizosphere microbial diversity naturally significantly increases, and the community structure becomes more complex.

Biotic and abiotic factors are assumed to influence the structural and functional diversity of the microbial communities in the rhizosphere (47-48). Site properties, including soil type, climatic conditions and type of agricultural management, have been shown to strongly influence the relative composition of rhizosphere microbial communities (48-54). Changes in the physical and chemical properties of soil also have a significant impact on fungal diversity and community structure in crop rhizospheres. Soil physical and chemical properties are determined not only by the nature of the soil itself but also by the physical and chemical properties of the soil after the interaction between crop roots and rhizosphere fungi. The physical and chemical soil properties we measured were determined in soil collected around the rhizosphere of wheat. Organisms that are present in the rhizosphere microbiota can have profound effects on the growth, nutrition and health of plants in agroecosystems $(25,55-57)$. TP and TC decreased from the heading stage to the filling stage, indicating that the later the growth period was, the less TP and TC was needed; however, these metrics seemed unrelated to the occurrence of diseases. In addition, the levels of $\mathrm{NH}_{4}, \mathrm{NO}_{3}$ and $\mathrm{TN}$ in the diseased group were higher than those in the healthy group at the heading and filling stages, indicating that their increase contributed to the occurrence of wheat root rot disease. In contrast, the $\mathrm{pH}$ and SD of the soils around diseased plants were lower than those of the soils around healthy plants at the heading and filling stages. This finding indicated that relatively low $\mathrm{pH}$ and SD values were beneficial to the occurrence of wheat root rot disease. The heatmap cluster analysis of the physical and chemical soil indicators and the 30 most abundant genera also showed that soil $\mathrm{pH}$ and SD affected fungal abundance and diversity in the same direction.

\section{Conclusions}

In the rhizosphere, many plant-microbial interactions occur that mediate soil processes (25). The occurrence of crop root rot disease is closely related to the interaction between rhizosphere microorganisms and crop roots, as well as the physical and chemical properties of soil. At present, 
there are approximately 10 types of fungi that can cause wheat root rot disease alone or in a complex, according to the literature. By studying the diversity of fungi and the community structure of the rhizospheres of healthy and diseased wheat at different growth stages, the heading and filling stages, it was revealed that in the early stages of illness, the high diversity of rhizosphere fungi and a complex community structure can easily cause wheat root rot disease. Additionally, the existence of pathogenic fungi is a necessary condition for wheat root rot disease, but the richness of pathogenic fungi is not necessarily important. Based on the physical and chemical properties of the soil, an increase in $\mathrm{NH}_{4}, \mathrm{NO}_{3}$ and $\mathrm{TN}$ contributes to the occurrence of wheat root rot disease. Soil $\mathrm{pH}$ and SD had the greatest influence on the abundance and diversity of rhizosphere fungi, and the influence was in the same direction; low soil $\mathrm{pH}$ and SD are beneficial to the occurrence of wheat root rot disease.

\section{Additional files}

Additional file 1: Supplemental figures (PDF $1838 \mathrm{~kb}$ )

Additional file 2: Supplemental tables (PDF $268 \mathrm{~kb}$ )

\section{ACKNOWLEDGEMENTS}

We thank L.J. Yang for insightful discussions; M.F. Xue and S.J. Gong for critical comments on the manuscript and J.Z. Ruan for land planted with wheat.

\section{FUNDING}

This work was

financially supported by the National Special Fund for Agro-scientific Research in the Public Interest of China (201503 112-8), the National Key Research and Development Program of China (2017YFD0200605), and t he Key Research and Development Project (2017YFD0201600).

\section{Avilability of data and materials}

All data generated during this study is available at the Sequence Read Archive (SRA) under BioProject number PRJNA549031.

\section{AUTHOR CONTRIBUTIONS}

W. H., and Y.D.Z. designed research; Z.X.J., and W.H. performed research; Z.X.J., W.H.Y., and A.G. analyzed data; and Z.X.J., W.H., and W.H.Y. wrote the manuscript with input from all authors. 


\section{Ethics approval and consent to participate}

Not applicable

\section{Consent for publication}

Not applicable.

\section{Competing interests}

The authors declare that they have no competing interests.

\section{Author details}

${ }^{1}$ Institute of Plant Protection and Soil \& Fertilizer, Hubei Academy of Agricultural Sciences/Key Laboratory of Integrated Pest Management on Crops in Central China, Ministry of Agriculture. P. R. China/Hubei Key Laboratory of Crop Disease, Insect Pests and Weeds Control, Wuhan 430064, P. R. China; ${ }^{2}$ HuBei University of Technology, Wuhan 430068, P. R. China; ${ }^{3}$ The Centre for Crop Health, University of Southern Queensland, Toowoomba QLD 4350, Australia.

\section{References}

1. Lupwayi, N.Z., Rice, W.A., and Clayton, G.W. Soil microbial diversity and community structure under wheat as influenced by tillage and crop rotation. Soil Biol. Biochem. 1998; 30(13):1733-41. 2. Parkinson, D., and Coleman, D.C. Microbial communities, activity and biomass. Agriculture, Ecosystems and Environment. 1981; 34:3-33.

3. Kumar, A., Kumar, A., and Pratush, A. Molecular diversity and functional variability of environmental isolates of Bacillus species. Springer Plus. 2014; 3:2-11.

4. Rashida, M.I., Mujawara, L.H., Shahzade, T., Almeelbi, T.I., Iqbal M.I., and Oves, M. Bacteria and fungi can contribute to nutrients bioavailability and aggregate formation in degraded soils. Microbiological Research. 2016; 183: 26-41.

5. Schreiner, R.P., and Bethlenfalvay, G.J. Mycorrhizae, biocides, and biocontrol. 4. Response of a mixed culture of arbuscular mycorrhizal fungi and host plant to three fungicides. Biology and Fertility of Soils. 1996; 23:189-95.

6. Assaf, T.A., Turk, M.A., and Ameed, K.M. Impact of olive pomace wastes and fungicide treatment on indigenous arbuscular mycorrhizal fungi associated with chickpea (Cicerarietinum L.) under field conditions. Australian Journal of Crop Science. 2009; 3:6-12.

7. Taheri, A.E., Hamel, C., and Gan Y.T. Pyrosequencing reveals the impact of foliar fungicide application to chickpea on root fungal communities of durum wheat in subsequent year. Fungal 
ecology. 2015; 15:73-81.

8. Ai, C., Liang, G.Q., Sun, J.W., Wang, X.B., He, P., Zhou, W., and He, X.H. Reduced dependence of rhizosphere microbiome on plant-derived carbon in 32-year long-term inorganic and organic fertilized soils. Soil Biology and Biochemistry. 2015; 80:70-8.

9. Kirkegaard, J., Christen, O., Krupinsky, J., and Layzell, D. Break crop benefits in temperate wheat production. Field Crops Res. 2008; 107:185-195.

10. Wintera, M., Mol, F.D., and Tiedemann, A.V. Cropping systems with maize and oilseed rape for energy production may reduce the risk of stem base diseases in wheat. Field Crops Research. 2014; 156:249-57.

11. Sivasithamparam, K. Ecology of root-infecting pathogenic fungi in Mediterranean environments. Adv. Plant Pathol. 1993; 10:245-79.

12. Almasudy, A.M., You, M.P., and Barbetti, M.J. Influence of fungicidal seed treatments and soil type on severity of root disease caused by Rhizoctonia solani AG-8 on wheat. Crop Protection. 2015; 75:40-5.

13. Duffy, B. Combination of pencycuron and pseudomonas yuorescens strain 2-79 for integrated control of rhizoctonia root rot and take-all of spring wheat. Crop Protection. 200; 19:21-5.

14. Paulitz, T.C., Smiley, R.W., and Cook, R.J. Insights into the prevalence and management of soilborne cereal pathogens under direct seeding in the Pacific Northwest, USA. Canadian Journal of Plant Pathology. 2002; 24:416-428.

15. Mavrodi, O.V., Walter, N., Elateek, S., Taylor, C.G., and Ubara P.A.O. Suppression of Rhizoctonia and Pythium root rot of wheat by new strains of Pseudomonas. Biological Control. 2012; 62:93-102.

16. Okubara, P.A., Dickman, M.B., and Blechl, A.E. Molecular and genetic aspects of controlling the soilborne necrotrophic pathogens Rhizoctonia and Pythium. Plant Science. 2014; 228:61-70.

17. Mielke, H. Studien zum Befall des Weizens mit Gaeumannomyces graminis (Sacc.) von Arx et Olivier var. tritici Walker unter Berücksichtigung der Sortenund Artenanfäligkeit sowie der Bekäpfung des Erregers. Mitteilungen aus der Biologischen Bundesanstalt für Land-und Forstwirtschaft, Berlin-Dahlem. Heft, pp. 1998; 359.

18. Paulitz, T.C., and Adams, K. Composition and distribution of Pythium communities in wheat fields in eastern Washington state. Phytopathology. 2003; 93:867-873.

19. Smith, J.D, Kidwell, K., Evans, M., Cook, R., and Smiley, R.W. Assessment of spring wheat genotypes for disease reaction to Rhizoctonia solani AG-8 in controlled environment and direct-seeded field evaluations. Crop Sci. 2003; 43:694-700.

20. Smith, J.D., Kidwell, K.K., Evans, M.A., Cook, R.J., and Smiley, R.W. Evaluation of spring cereal grains and wild Triticum relatives for resistance to Rhizoctonia solani AG 8. Crop Sci. 2002; 43:701-709.

21. Davis, R.A., Huggins, D., Cook, R.J., and Paulitz, T.C. Can placement of seed away from relic stubble limit Rhizoctonia root rot in direct-seeded wheat? Soil and Tillage Research. 2008; 101:37- 
43.

22. Paulitz, T.C., and Scott, R.B. Effect of Seed Treatments for Control of Rhizoctonia Root Rot in Spring Wheat, 2005. Fungicide and Nematicide Reports. 2006; 61:ST014.

23. Meena, V.S., Maurya, B.R., Bohra, J.S., Verma, R., and Meena, M.D. Effect of concentrate manure and nutrient levels on enzymatic activities and microbial population under submerged rice in alluvium soil of Varanasi. Crop Res. 2013; 45 (1,2 and 3):6-12.

24. Li, M., Ahammed, G.J., Li, C., Bao, X., Yu, J., Huang, C., Yin, H., and Zhou, J. Brassinosteroid ameliorates zinc oxide nanoparticles-induced oxidative stress by improving antioxidant potential and redox homeostasis in tomato seedling. Front. Plant Sci. 2016; 7:615.

25. Kumar, A., Maurya, B.R., Raghuwanshi, R., Meena, V.S., and Islam, M.T. Co-inoculation with Enterobacter and Rhizobacteria on yield and nutrient uptake by wheat (Triticum aestivum L.) in the alluvial soil under the Indo-Gangetic Plain of India. J. Plant Growth Regul. 2017;

http://dx.doi.org/10.1007/s00344-016-9663-5

26. Kristin, A., and Miranda, H. The root microbiota - a fingerprint in the soil? Plant Soil. 2013; 370:671-686.

27. Lakshmanan, V., Selvaraj, G., and Bais, H.P. Functional soil microbiome: below ground solutions to an aboveground problem. Plant Physiol. 2014; 166:689-700.

28. Miao, C.P., Mi, Q.L., Qiao, X.G., Zheng, Y.K., Chen, Y.W., Xu, L.H., Guan, H.L., and Zhao, L.X. Rhizospheric fungi of Panax notoginseng: diversity and antagonism to host phytopathogens. J Ginseng Res. 2016; 40:127-134.

29. Chakraborty, U., Swarnendu, R., Pratim, C.A., Pannalal, D., and Bishwanath, C. Plant growth promotion and amelioration of salinity stress in crop plants by a salt-tolerant bacterium. Recent Res. Sci. Technol. 2011; 3 (11):61-70.

30. Meena, S.K., Rakshit, A., and Meena, V.S. Effect of seed bio-priming and N doses under varied soil type on nitrogen use efficiency (NUE) of wheat (Triticumaestivum L.) under greenhouse conditions. Biocata. Agric. Biotechnol. 2016; 6:68-75.

31. Narula, S., Anand, R.C., and Dudeja, S.S. Beneficial traits of endophytic bacteria from field pea nodules and plant growth promotion of field pea. J. Food Legumes. 2013; 26:73-79.

32. Ramırez-Bahena, M.H., Tejedor, C., Martín, I., Velaíquez, E., and Peix, A. Enterobacter medicaginis gen. nov. sp. nov., isolated from alfalfa nodules in anacidic soil. Int. J. Syst. Evol. Microbiol. 2013;63:1760-1765.

33. Handiseni, M., Brow, J., Zemetra, R., and Mazzol, M. Effect of Brassicaceae seed meals with different glucosinolate profiles on Rhizoctonia root rot in wheat. Crop Protection. 2013; 48: 1-55. 34. Phillips, R., and Fahey, T. Fertilization effects on fine root biomass, rhizosphere microbes and respiratory fluxes in hardwood forest soils. New Phytologist. 2007; 176:655-664.

35. Buyer, J.S., Roberts, D.P., and Russek-Cohen, E. Microbial community structure and function in the spermosphere as affected by soil and seed type. Can J Microbiol. 1999; 45:138-144.

36. Rasche, F., Hödl, V., Poll, C., Kandeler, E., Gerzabek, M.H., van Elsas, J.D., and Sessitsch, A. 
Rhizosphere bacteria affected by transgenic potatoes with antibacterial activities compared with the effects soil, wild-type potatoes, vegetation stage and pathogen exposure. FEMS Microbiol Ecol. 2006; 56:219-235.

37. Söderberg, K.H., Olsson, P.A., and Bååth, E. Structure and activity of the bacterial community in the rhizosphere of different plant species and the effect of arbuscular mycorrhizal colonisation. FEMS Microbiol Ecol. 2002; 40:223-231.

38. Gyamfi, S., Pfeifer, U., Stierschneider, M., and Sessitsch, A. Effects of transgenic glufosinate-tolerant oilseed rape (Brassica napus) and the associated herbicide application on eubacterial and pseudomonas communities in the rhizosphere. FEMS Microbiol Ecol. 2002; 41:181-190.

39. Dunfield, K.E., and Germida, J.J. Seasonal changes in the rhizosphere microbial communities associated with field-grown genetically modified canola (Brassica napus). Appl. Environ. Microbiol. 2003; 69:7310-7318.

40. Houlden, A., Timms-Wilson, T.M., Day, M.J., and Bailey, M.J. Influence of plant developmental stage on microbial community structure and activity in the rhizosphere of three field crops. FEMS Microbiol. Ecol. 2008; 65:193-201.

41. Li, X.Z., Rui, J.P., Mao, Y.J., Yannarell, A., and Mackie, R. Dynamics of the bacterial community structure in the rhizosphere of a maize cultivar. Soil Biology and Biochemistry. 2014; 68: 392-401.

42. Xu, L., Ravnskov, S., Larsen, J., Nilsson, R.H., Nicolaisen, M. Soil fungal community structure along a soil health gradient in pea fields examined using deep amplicon sequencing. Soil Boil Biochem. 2012; 46:26-32.

43. Mendes, R., Garbeva, P., and Raaijmakers, J.M. The rhizosphere microbiome: significance of plant beneficial, plant pathogenic, and human pathogenic microorganisms. FEMS Microbiol Rev. 2013; 37:634-663.

44. Singh, A.K., Singh, M., and Dubey, S.K. Rhizospheric fungal community structure of a Bt brinjal and a near isogenic variety. J Appl Microbiol. 2014; 117:750-765.

45. Buée, M., Reich, M., Murat, C., Morin, E., Nilsson, R.H., Uroz, S., and Martin, F. 454 Pyrosequencing analyses of forest soils reveal an unexpectedly high fungal diversity. New Phytol. 2009; 184:449-456.

46. Hayat, R., Ali, S., Amara, U., Khalid, R., and Ahmed, I. Soil beneficial bacteria and their role in plant growth promotion: a review. Ann Microbiol. 2010; 60:579-598.

47. Berg, G., and Smalla, K. Plant species and soil type cooperatively shape the structure and function of microbial communities in the rhizosphere. FEMS Microbiol Ecol. 2009; 68:1-13. 48. Weinert, N., Piceno, Y., Ding, G.C., Meincke, R., Heuer, H., and Berg, G. PhyloChip hybridization uncovered an enormous bacterial diversity in the rhizosphere of di $\square$ rent potato cultivars: many common and few cultivar-dependent taxa. FEMS Microbiol Ecol. 2011; 75:497506. 
49. Heuer, H., Kroppenstedt, R.M., Lottmann, J., Berg, G., and Smalla, K. Effects of T4 lysozyme release from transgenic potato roots on bacterial rhizosphere communities are negligible relative to natural factors. Appl Environ Microbiol. 2002; 68(3):1325-1335.

50. Kowalchuk, G.A., Buma, D.S., de Boer, W., Klinkhamer, P.G.L., and van Veen, J.A. Effects of above-ground plant species composition and diversity on the diversity of soil-borne microorganisms. Antonie van Leeuwenhoek. 2002; 81:509-520.

51. Berg, G., Opelt, K., Zachow, C., Lottmann, J., Götz, M., Costa, R., and Smalla, K. The rhizosphere effect on bacteria antagonistic towards the pathogenic fungus Verticillium differs depending on plant species and site. FEMS Microbiol Ecol. 2006; 56:250-261.

52. Costa, R., Götz, M., Mrotzek, N., Lottmann, J., Berg, G., and Smalla, K. Effects of site and plant species on rhizosphere community structure as revealed by molecular analysis of microbial guilds. FEMS Microbiol Ecol. 2006; 56:236-249.

53. Bremer, C., Braker, G., Matthies, D., Reuter, A., Engels, C., and Conrad, R. Impact of plant functional group, plant species, and sampling time on the composition of nirK-type denitrifier communities in soil. Appl Environ Microb. 2007; 73:6876-6884.

54. Weinert, N., Meincke, R., Gottwald, C., Radl, V., Dong, X., Schloter M., Berg, G., and Smalla K. Effects of genetically modified potatoes with increased zeaxanthin content on the abundance and diversity of rhizobacteria with in vitro antagonistic activity do not exceed natural variability among cultivars. Plant Soil. 2010; 326:437-452.

55. Bonfante, P., and Anca, I.A. Plants, mycorrhizal fungi, and bacteria: a network of interactions. Annu. Rev. Microbiol. 2009; 63:363-383.

56. Mendes, R., Kruijt, M., De Bruijn, I., Dekkers, E., van der Voort, M., Schneider, J.H.M., Piceno, Y.M., DeSantis T.Z., Andersen G.L., Bakker P.A.H.M., and Raaijmakers J.M. Deciphering the rhizosphere microbiome for disease-suppressive bacteria. Science. 2011; 332:1097-1100.

57. Meena, R.S., Meena, V.S., Meena, S.K., and Verma, J.P. The needs of healthy soils for a healthy world. J. Cleaner Prod. 2015; 102:560-561.

\section{FIGURES}




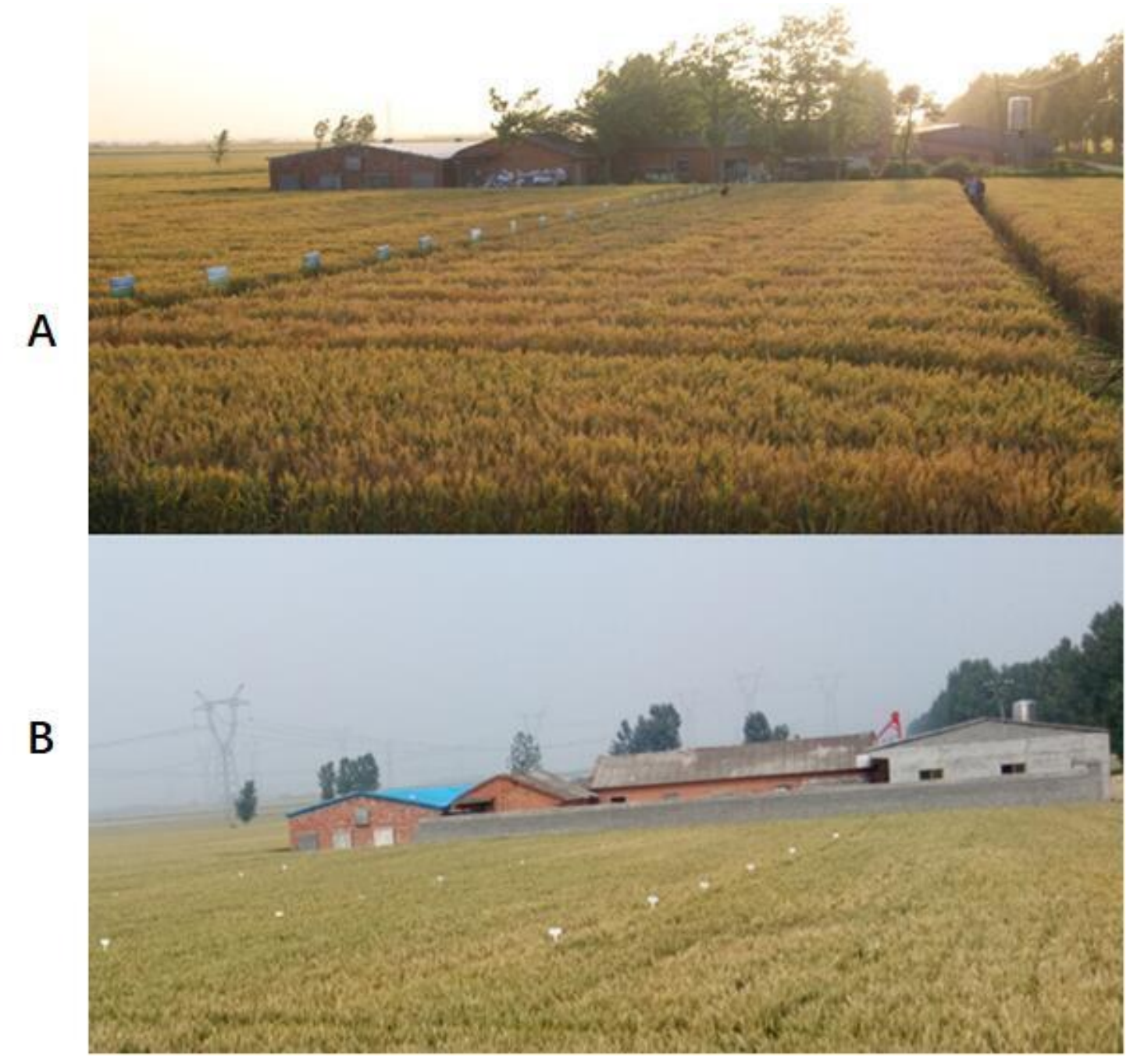

Fig. 1 Sampling plots of wheat root. a The occurrence of wheat root rot in this wheat field in 2015 and 2016. b The soil sample collection of wheat root in this wheat field in 2017. Areas with diseased wheat and healthy wheat were marked by inserting white cards at fixed points. The samples, in April and May, were collected from the areas labeled in April. 

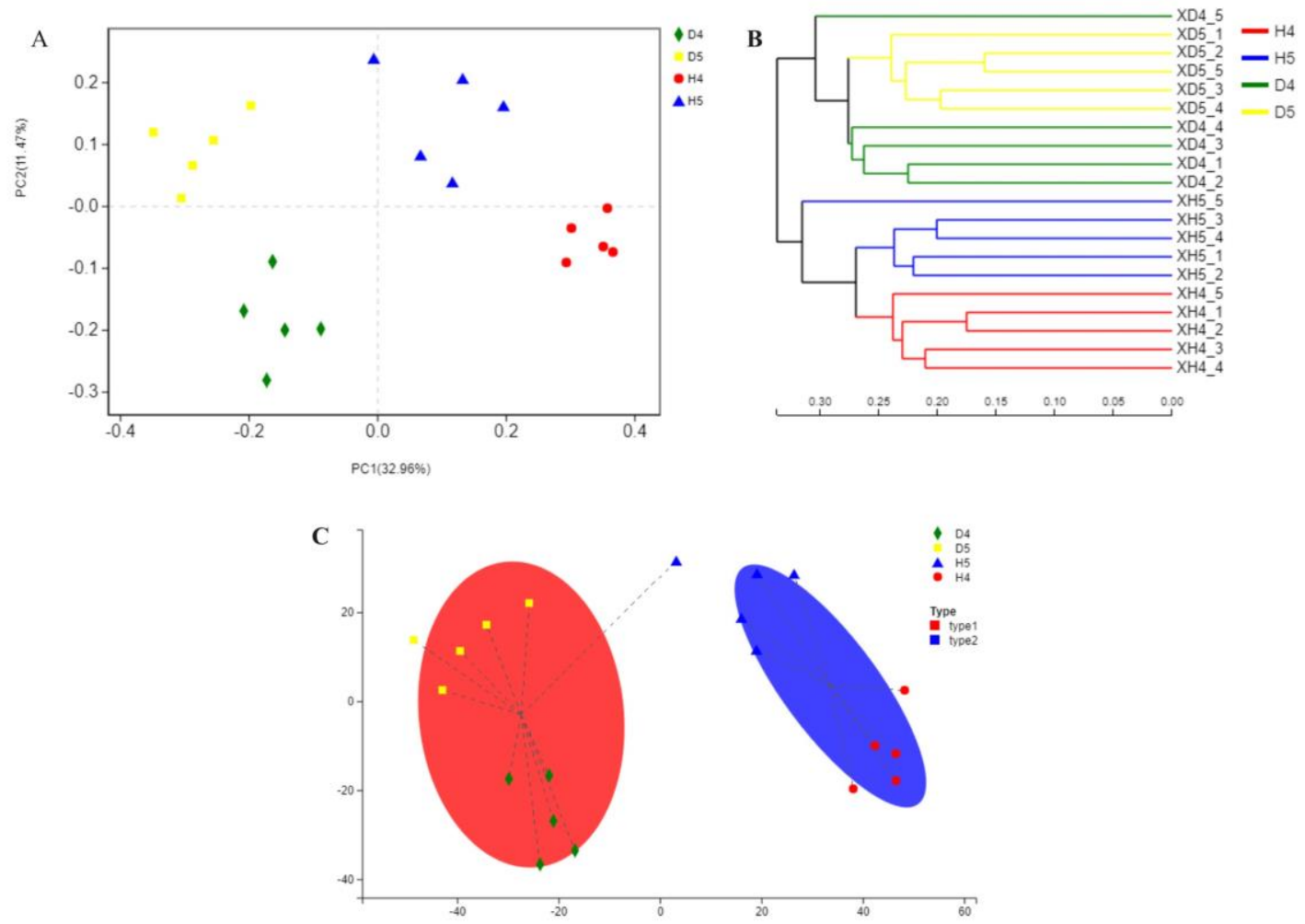

Fig. 2 Comparative analysis of OTUs and genus levels in each community. a Principal coordinate analysis (PCoA) at the OTU level in healthy and diseased groups at the heading stage and filling stage. Distance algorithm is based on bray_curtis. b Sample hierarchical clustering analysis based on OTU level. $\mathbf{c}$ Typing analysis of fungi at the genus level. 

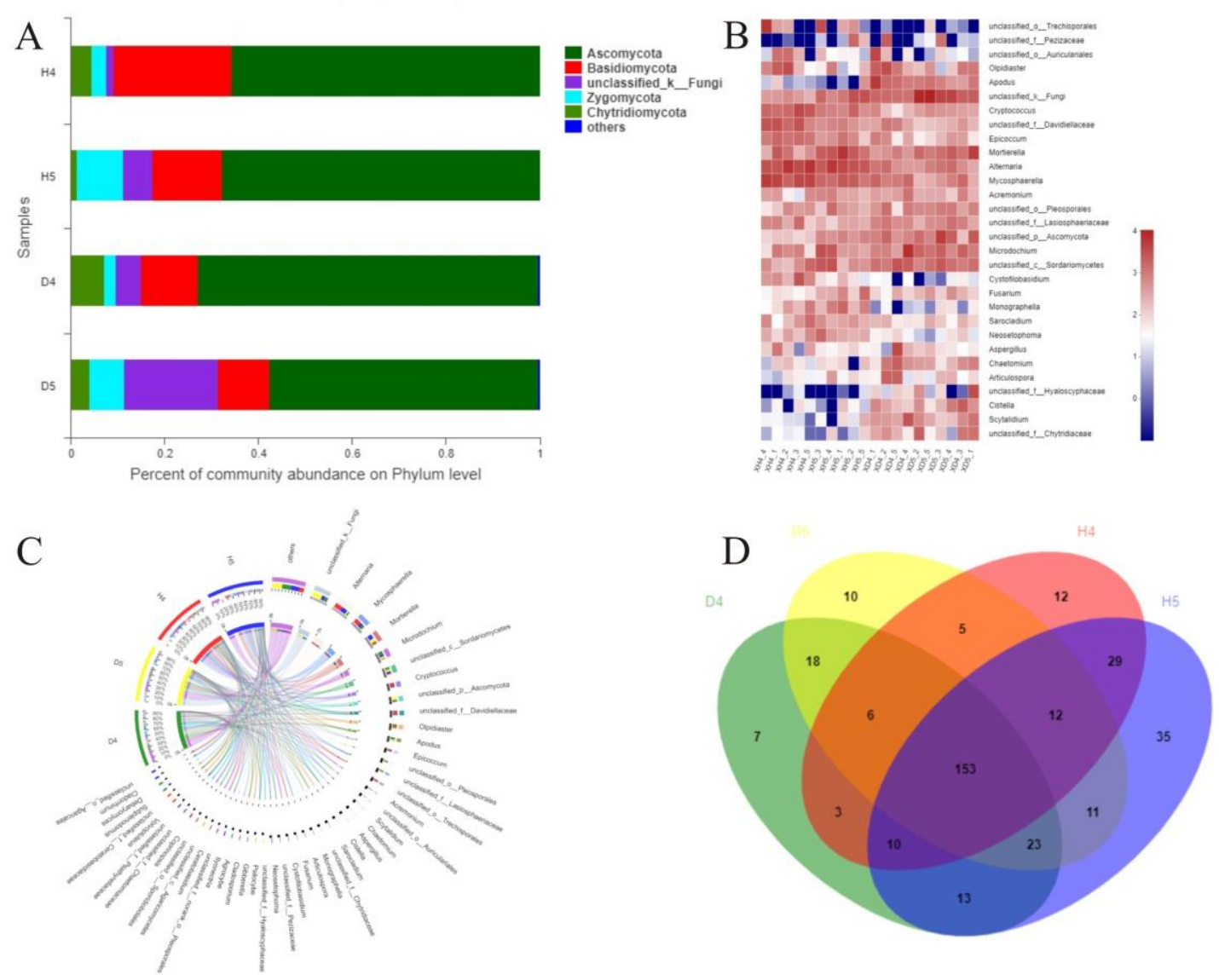

Fig. 3 Species composition analysis. a Percent community abundance at the phylum level in each groups. b Species abundance clustering at genus level in each sample. c Circos representation showing distribution of genus with significant difference in abundance in different groups. $\mathbf{d}$ Analysis of common and endemic genera in the different populations by Venn diagram. 


\section{Spearman Correlation Heatmap}

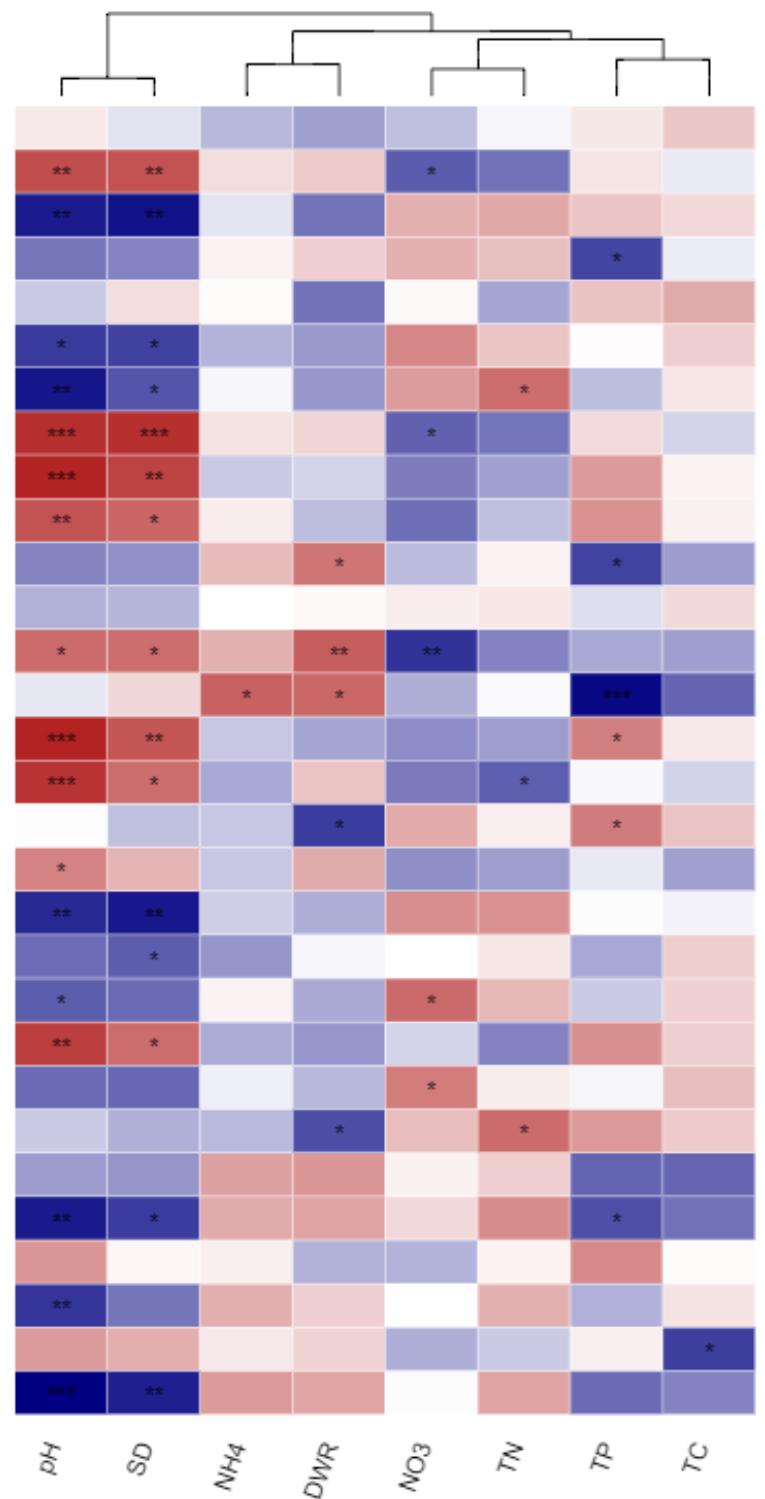

Acremonium

Alternaria

Apodus

Articulospora

Aspergillus

Chaetomium

Cistella

Cryptococcus

Cystofilobasidium

Epicoccum

Fusarium

Microdochium

Monographella

Mortierella

Mycosphaerella

Neosetophoma

Olpidiaster

Sarocladium

Scytalidium

unclassified_c__Sordariomycetes

unclassified_f__Chytridiaceae

unclassified_f__Davidiellaceae

unclassified_f__Hyaloscyphaceae

unclassified_f__Lasiosphaeriaceae

unclassified_f__Pezizaceae

unclassified_k__Fungi

unclassified_0__Auriculariales

unclassified_o__Pleosporales

unclassified_0__Trechisporales

unclassified_p_Ascomycota

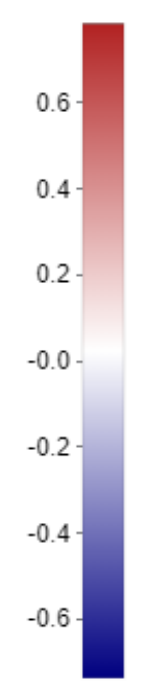

Fig. 4 Hierarchical clustering analysis at the OTU level between physical and chemical soil properties and the 30 most abundant genera. 


\section{TABLES}

Table 1 The analysis of alpha diversity index in healthy and diseased groups.

\begin{tabular}{|l|l|l|l|l|l|}
\hline SamplelEstimators & Sobs & Ace & Chao & Shannon & Coverage \\
\hline H4 & 129.40 & 142.21 & 142.14 & 3.03 & 0.9994 \\
\hline D4 & 135.60 & 154.71 & 149.52 & 3.37 & 0.9995 \\
\hline H5 & 163.40 & 178.98 & 180.19 & 3.41 & 0.9993 \\
\hline D5 & & & & & \\
\hline
\end{tabular}


Table 2 All fungi with a richness greater than one percent in each group. There are 30 genera in the four groups, which mainly belong to Ascomycota, Basidiomycota, and Chytridiomycota.

\begin{tabular}{|c|c|c|c|c|c|}
\hline Phylum & Genus & $\mathrm{H} 4$ & D4 & H5 & D5 \\
\hline \multirow[t]{21}{*}{$p_{\text {__Ascomycota }}$} & g___Microdochium & 0.0184 & 0.0760 & 0.0455 & 0.0454 \\
\hline & g__Apodus & 0.0010 & 0.0662 & 0.0035 & 0.0314 \\
\hline & g__Mycosphaerella & 0.1291 & 0.0460 & 0.0609 & 0.0136 \\
\hline & $g \_$Scytalidium & 0.0005 & 0.0309 & 0.0027 & 0.0168 \\
\hline & $g \_$Chaetomium & 0.0028 & 0.0295 & 0.0029 & 0.0132 \\
\hline & g__Alternaria & 0.1489 & 0.0284 & 0.1160 & 0.0288 \\
\hline & g_Aspergillus & 0.0119 & 0.0274 & 0.0021 & 0.0035 \\
\hline & g_Articulospora & 0.0012 & 0.0271 & 0.0044 & 0.0045 \\
\hline & $g \_$Cistella & 0.0010 & 0.0236 & 0.0015 & 0.0164 \\
\hline & g_Acremonium & 0.0103 & 0.0213 & 0.0165 & 0.0081 \\
\hline & g__Epicoccum & 0.0442 & 0.0160 & 0.0201 & 0.0057 \\
\hline & g__Subplenodomus & 0.0005 & 0.0134 & 0.0009 & 0.0001 \\
\hline & g__Debaryomyces & 0.0008 & 0.0108 & 0.0001 & 0.0003 \\
\hline & g__Sarocladium & 0.0193 & 0.0055 & 0.0127 & 0.0042 \\
\hline & g_Fusarium & 0.0021 & 0.0050 & 0.0197 & 0.0100 \\
\hline & g__Gibberella & 0.0122 & 0.0036 & 0.0083 & 0.0038 \\
\hline & g__Cladosporium & 0.0135 & 0.0027 & 0.0103 & 0.0013 \\
\hline & g__Neosetophoma & 0.0106 & 0.0025 & 0.0195 & 0.0008 \\
\hline & g___Monographella & 0.0114 & 0.0003 & 0.0259 & 0.0015 \\
\hline & g__llyonectria & 0.0025 & 0.0001 & 0.0210 & 0.0005 \\
\hline & g__Cladorrhinum & 0.0000 & 0.0000 & 0.0118 & 0.0000 \\
\hline$p_{p \_}$Basidiomycota & g_Agrocybe & 0.0000 & 0.0255 & 0 & 0.0006 \\
\hline
\end{tabular}




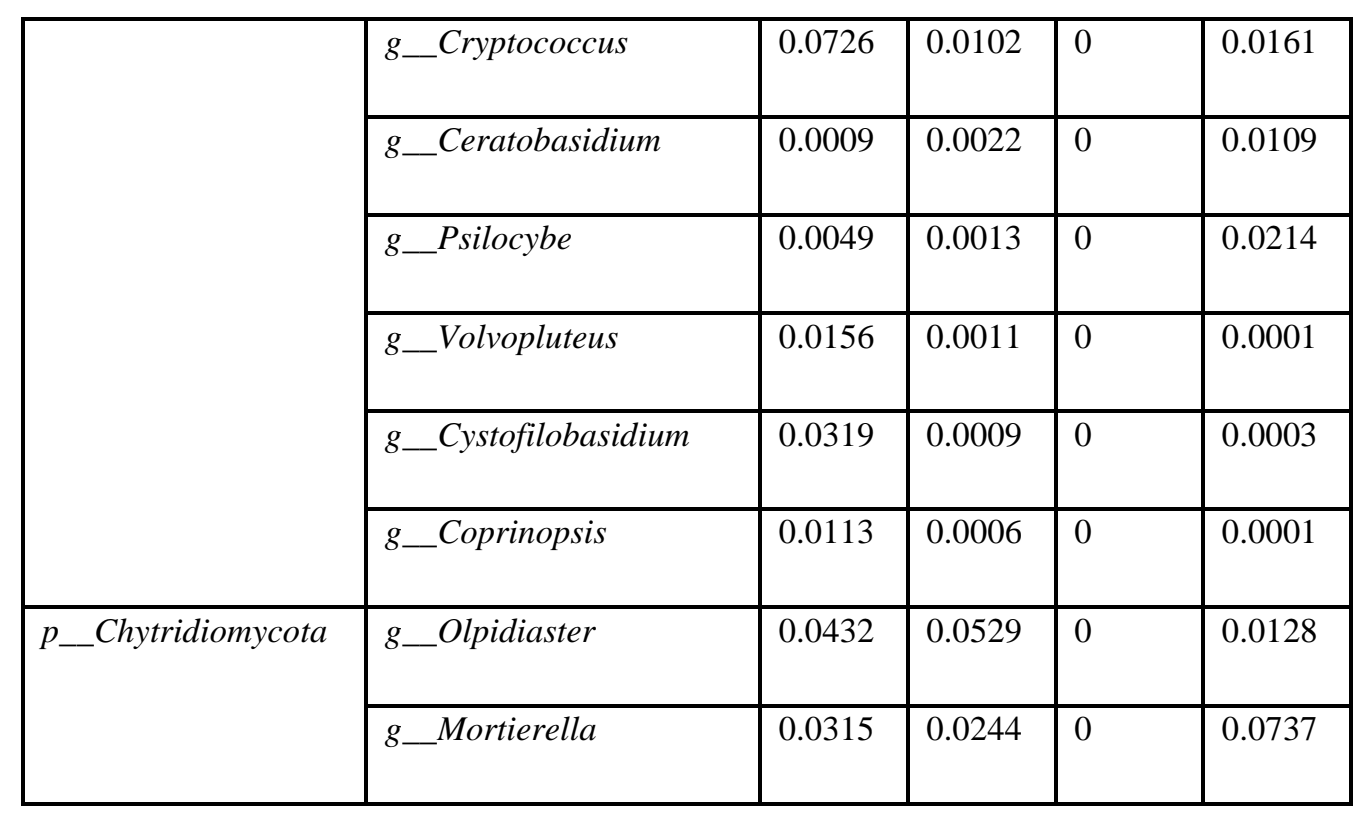


Table 3 The physical and chemical properties of the rhizosphere.

\begin{tabular}{|l|l|l|l|l|l|l}
\hline Sample ID & TP & $\mathrm{NH}_{4}$ & $\mathrm{NO}_{3}$ & $\mathrm{TN}$ & $\mathrm{pH}$ & $\mathrm{SD}$ \\
\hline $\mathrm{CH} 4$ & $4.16 \pm 0.13$ & $16.02 \pm 3.27$ & $26.38 \pm 6.03$ & $204.1 \pm 30.94$ & $7.18 \pm 0.06$ & $2.68 \pm 0.06$ \\
\hline CD4 & $4.13 \pm 0.21$ & $14.04 \pm 2.62$ & $30.97 \pm 5.95$ & $251.48 \pm 21.09$ & $6.95 \pm 0.08$ & $2.42 \pm 0.05$ \\
\hline CH5 & $3.82 \pm 0.18$ & $17.45 \pm 4.20$ & $23.48 \pm 2.23$ & $217.65 \pm 49.43$ & $7.15 \pm 0.07$ & $2.5 \pm 0.03$ \\
\hline CD5 & $3.84 \pm 0.16$ & $18.71 \pm 2.41$ & $29.38 \pm 3.82$ & $237.39 \pm 52.74$ & $6.76 \pm 0.15$ & $2.48 \pm 0.03$ \\
\hline
\end{tabular}

*TP: total phosphorus, $\mathrm{NH}_{4}$ : ammonium nitrogen, $\mathrm{NO}_{3}$ : nitrate nitrogen, $\mathrm{TN}$ : total nitrogen, $\mathrm{SD}$ : soil density, TC: total carbon, DHR: soil dry-humidity ratio. 